BULLETIN (New Series) OF THE AMERICAN MATHEMATICAL SOCIETY

Volume 35, Number 3, July 1998, Pages 193-214

S 0273-0979(98)00750-2

\title{
GEOMETRY OF RIEMANN SURFACES BASED ON CLOSED GEODESICS
}

\author{
PAUL SCHMUTZ SCHALLER
}

\begin{abstract}
The paper presents a survey on recent results on the geometry of Riemann surfaces showing that the study of closed geodesics provides a link between different aspects of Riemann surface theory such as hyperbolic geometry, topology, spectral theory, and the theory of arithmetic Fuchsian groups. Of particular interest are the systoles, the shortest closed geodesics of a surface; their study leads to the hyperbolic geometry of numbers with close analogues to classical sphere packing problems.
\end{abstract}

\section{Contents}

1. Introduction

2. Basic definitions

3. Hyperelliptic surfaces and Weierstrass points

4. Hyperbolic geometry of numbers

5. Systole as a Morse function

6. Geometric characterization of arithmetic Fuchsian groups and the multiplicity in the length spectrum

7. Simple closed geodesics of the once-punctured torus and the Markoff spectrum

8. Small eigenvalues of the Laplacian

References

\section{INTRODUCTION}

Riemann surfaces are basic mathematical objects. Many important mathematical theories are related to Riemann surfaces. This contributes to the permanent renewing of Riemann surface theory and keeps this theory vital. In this article Riemann surfaces are presented from a geometric point of view, based on hyperbolic geometry, where closed geodesics in general and simple closed geodesics in particular play a crucial role.

Riemann surface theory based on (simple) closed geodesics has proved to be fruitful due to its natural relation to other approaches. First of all there is an obvious relation with topology. This goes back to Riemann himself, who, in his Inauguraldissertation [78], introduced and defined the genus of a surface $M$ to be the maximal number of disjoint simple closed curves along which one can cut $M$, such that the resulting surface is still connected. The topological aspects of simple

Received by the editors October 1, 1997, and, in revised form, March 19, 1998.

1991 Mathematics Subject Classification. Primary 30F45, 53C22, 57M50, 11F06, 11H99; Secondary $32 \mathrm{G} 15,11 \mathrm{~F} 72$.

Partially supported by Schweizerischer Nationalfonds. 
closed curves were further developed by Dehn [26], Nielsen [70] and, in the seventies, by Thurston; see [98],[99],[31],[17],[19],,[74],[10],[11],[56].

Of similar importance is the relation of lengths of closed geodesics of a Riemann surface with traces of elements of a corresponding Fuchsian group. An important consequence is that the arithmetic Fuchsian groups can be characterized geometrically by the lengths of closed geodesics; implicitly, this goes back to Takeuchi [97] (see section 6 for more on this subject). As one of the consequences many results and problems in classical number theory can be expressed by a geometric language using lengths of closed geodesics. For example, in Fricke/Klein [34] the classical theory of indefinite binary quadratic forms was, in parts, reformulated using the modular group and the traces of its elements (which are, as we have just seen, closely related to lengths of closed geodesics). Or the Gauss conjecture stating that there are infinitely many different real quadratic number fields of class number one can be expressed in terms of lengths of closed geodesics; see [88]. A particularly nice connection between simple closed geodesics and number theory is described in section 7 for once-punctured tori.

There is a long tradition of putting concepts of the (Euclidean) geometry of numbers into the context of hyperbolic geometry (an early example is Delsarte [27]). But only quite recently it turned out (this started with Schmutz [82]; see section 4) that the most natural objects in this context are the systoles, the shortest closed geodesics of a Riemann surface. They provide perfect analogues to classical problems of lattice sphere packings. An important fact (see section 5) is also that the function syst which associates to every surface the length of its systole is a topological Morse function on Teichmüller space, equivariant with respect to the mapping class group ([90]), again in analogy to the packing function for lattice sphere packings (Ash [4]).

A very important relation is that provided by Selberg's trace formula. It states (in a quantitative form) that, for hyperbolic surfaces of finite area, the eigenvalue spectrum of the Laplacian determines the length spectrum and vice versa (see [93]); for closed surfaces the result was also proved independently by Huber [45]. Section 8 describes one aspect of the relation between closed geodesics and Laplacian eigenvalues.

The relation of closed geodesics to complex analysis is not yet very well developed. One may consider the geometric characterization of hyperelliptic Riemann surfaces [89],[91] as a subject in this context; see section 3. I also mention the geometric description of the Weil-Petersson metric on Teichmüller space; see [106],[107],[10].

There is a well known relation to ergodic theory, for example through the geodesic flow or through symbolic dynamics. This theory is not treated in this paper. Also, the relation with the theory of 3-manifolds and with Kleinian groups is not treated here.

The explicit geometric theory, based on closed geodesics, has a long tradition. In Fricke/Klein [34] (Zweiter Abschnitt, Sechstes Kapitel, §11), the systoles of the unique closed surface of genus 3 with an automorphism group of order 168 were already identified. The celebrated Fenchel/Nielsen manuscript [33] was another rich source. Important progress was done in the eighties; in particular, the convex structure of Teichmüller space provided by geodesic length functions was discovered in work of Kerckhoff [52],[53] and Wolpert [108]. Much work on the geometry of 
Riemann surfaces has further been done by the "Swiss school of hyperbolic geometry", initiated by Huber, in particular in connection with Laplacian eigenvalues; this is documented in Buser [16].

The content of the different sections has already been sketched. Every section (except section 2, which gives the basic definitions) contains open questions in the form of problems or conjectures.

I apologize for not considering enough related work by other authors. This text is mainly based on my own work and, in particular, on the talk I gave on the occasion of Heinz Huber's 70th birthday (1996) in Basel (Switzerland) and on the talk at the 17th Nevanlinna colloquium (1997) in Lausanne (Switzerland). I have profited a great deal by discussions and by comments on an earlier version of this paper. I notably thank Peter Buser, Jozef Dodziuk, Henryk Iwaniec, Greg McShane, Akimou Ossé, Jack Quine, Igor Rivin, Zeév Rudnick, and Peter Sarnak. I finally thank very much the referee for valuable comments.

\section{BASIC DEFINITIONS}

In this section I give some basic definitions which will be used throughout the paper.

Definition. (i) A surface $M$ is a Riemann surface equipped with a metric of constant curvature $-1 ; g$ is the genus of $M$. A $(g, n)$-surface is a surface of genus $g$ with $n$ cusps.

(ii) $T_{g}$ denotes the Teichmüller space of surfaces of genus $g . T_{g, n}$ denotes the Teichmüller space of $(g, n)$-surfaces.

(iii) $L(u)$ or $L_{M}(u)$ denotes the length of a closed geodesic of a surface $M$. Sometimes, the quantity

$$
2 \cosh (L(u) / 2)
$$

is more convenient than $L(u)$; I shall call this quantity the norm of $u$.

(iv) Closed geodesics in surfaces of the same Teichmüller space are considered as marked. In this sense, for a closed geodesic $u$ in a $(g, n)$-surface, I define the length function

$$
L(u): T_{g, n} \longrightarrow \mathrm{R}_{+}
$$

where $L_{M}(u)$ denotes the length of $u$ in $M \in T_{g, n}$.

(v) A simple closed geodesic is a closed geodesic without self-intersections.

(vi) An embedded subsurface $M^{\prime}$ of a surface $M$ is called a pair of pants if $M^{\prime}$ has genus 0 and three boundary components which are simple closed geodesics or cusps.

(vii) A systole of a $(g, n)$-surface $M$ is a shortest closed geodesic of $M$.

(viii) A Fuchsian group is the image in $P S L(2, \mathrm{R})$ of a discrete subgroup of $S L(2, \mathrm{R})$.

(ix) Let $M$ be a surface. Then I also write $M$ as

$$
M=\mathrm{H} / \Gamma
$$

where $\mathrm{H}$ denotes the upper halfplane and $\Gamma$ is a Fuchsian group. I call $\Gamma$ a corresponding Fuchsian group.

Remark. (i) In this paper, all surfaces will have corresponding Fuchsian groups which are finitely generated.

(ii) A Teichmüller space in this paper is always a space $T_{g}$ or $T_{g, n}$. In particular, if $M$ and $M^{\prime}$ are two $(g, n)$-surfaces, then I identify the Teichmüller space of $M$ and the Teichmüller space of $M^{\prime}$. 
(iii) The topology and the analytic structure of Teichmüller space are given by a set of length functions.

(iv) Let $M$ be a surface and $\Gamma$ a corresponding Fuchsian group. To a closed geodesic $u$ in $M$ correspond conjugacy classes $\bar{\gamma}$ and $\overline{\gamma^{-1}}$ in $\Gamma$ (which may be equal), and we have the identity (for every $\gamma$ in these conjugacy classes)

$$
2 \cosh (L(u) / 2)=|\operatorname{tr}(\gamma)|
$$

where $t r$ stands for trace. Note that on the left hand side, we have the norm of $u$. (v) A standard reference for this material is Buser [16]; see also Abikoff [1], Imayoshi/Taniguchi [46], Seppälä/Sorvali [94].

Definition. (i) The modular group $\Gamma(1)$ is defined as $\Gamma(1)=S L(2, \mathrm{Z}) / \sim$ with $U \sim V(U, V \in S L(2, \mathrm{Z}))$ if and only if $U= \pm V$.

(ii) For a positive integer $N>2$ the principal congruence subgroup $\Gamma(N)$ of the modular group is defined as

$$
\Gamma(N)=\left\{\left[\begin{array}{cc}
1+a N & b N \\
c N & 1+d N
\end{array}\right] \in S L(2, \mathrm{Z}) \mid a, b, c, d \in \mathrm{Z}\right\} .
$$

$\Gamma(N)$ is canonically identified with its image in $\operatorname{PS} L(2, \mathrm{R})$.

In the case $N=2$, the definition is

$$
\Gamma(N)=\left\{\left[\begin{array}{cc}
1+a N & b N \\
c N & 1+d N
\end{array}\right] \in S L(2, \mathrm{Z}) \mid a, b, c, d \in \mathrm{Z}\right\} / \sim
$$

with $U \sim V$ if and only if $U= \pm V$.

\section{Hyperelliptic SURfaces and Weierstrass Points}

In the classical theory, hyperelliptic Riemann surfaces are considered as the simplest surfaces. As algebraic curves of genus $g$ they can be defined as

$$
y^{2}=\sum_{i=0}^{2 g+2} a_{i} x^{i} .
$$

Here, the exponent 2 in $y^{2}$ is the crucial point. One immediately notices the hyperelliptic involution given by

$$
(x, y) \longrightarrow(x,-y)
$$

which has $2 g+2$ fixed points (see for example [30],[67]). Hyperelliptic surfaces can be characterized geometrically by simple closed geodesics.

Theorem 1. (Schmutz Schaller [89],[91]) Let $M \in T_{g}$. Then $M$ is hyperelliptic if and only if $M$ has a set $S$ of (at least) $2 g-2$ simple closed geodesics which all intersect in the same point such that the elements of $S$ have no further intersection point.

One may illustrate this result as in Figure 1. The condition of a unique intersection point is a priori a local one, but one can imagine that if the number of simple closed geodesics is big enough, then the condition becomes global.

In order to prove Theorem 1, a further, purely topological result is needed.

Theorem 2. ([89]) Let $M \in T_{g}$. Let $k$ be an integer with $1 \leq k \leq 2 g$. Let $S_{k}$ be a set of $k$ simple closed curves which intersect (transversally) in the same point (such 


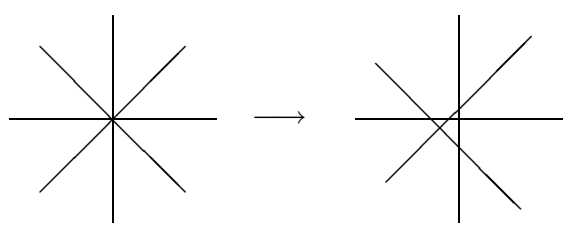

Figure 1. Four simple closed geodesics which intersect in the same point in the figure on the left hand side. A perturbation of the hyperbolic metric gives the figure on the right hand side.

that the elements of $S_{k}$ have no further intersection point). Then there is only one equivalence class of sets $S_{k}$ if and only if $k$ is even.

Here, $S_{k}$ and $S_{k}^{\prime}$ are in the same equivalence class if $M$ has a homeomorphism mapping the free homotopy classes of the elements of $S_{k}$ to those of $S_{k}^{\prime}$.

The idea of the proof of Theorem 2 is the fact that if $k$ is odd, then there exists a separating set $S_{k}$ (meaning that

$$
M \backslash \bigcup_{u \in S_{k}} u
$$

is not connected) as well as a non-separating set $S_{k}$ which of course cannot be in the same equivalence class. This is a generalization of the fact that there are separating and non-separating simple closed curves (this is the case $k=1$ ). On the other hand, if $k \leq 2 g$ is even, then $S_{k}$ is always non-separating.

Back to Theorem 1, note that $2 g-2$ is even and that therefore, the set of $2 g-2$ simple closed geodesics is topologically determined by Theorem 2 .

In the hyperelliptic case the intersection point in Theorem 1 is a fixed point of the hyperelliptic involution. It is also a Weierstrass point. Every surface in $T_{g}$ has between $2 g+2$ and $g^{3}-g$ different Weierstrass points (see for example [30],[67]). Theorem 1 gives a geometric description of the Weierstrass points in the hyperelliptic case, and one would like to generalize this result.

Problem. Find a geometric characterization (based on closed geodesics) of Weierstrass points.

\section{Hyperbolic GeOMETRY OF NUMBERS}

Let $T$ be a Euclidean torus (with Euclidean metric) in the plane. Let $F(T)$ be a parallelogram with vertices $A, B, C, D$ which is a fundamental domain for $T$. Let $u$ be a shortest closed geodesic of $T$ passing through two vertices of $F(T), A$ and $B$ say. Then $u$ defines a circle packing of the plane if one puts circles of radius $L(u) / 2$ centered in the vertices of the lattice defined by $F(T)$; see Figure 2. Vice versa, a lattice circle packing in the plane defines a torus and the length of its shortest closed geodesic. Therefore, a shortest geodesic of a Euclidean torus and a lattice circle packing in the plane are isomorphic concepts.

Now there are two different directions of a possible generalization. On the one hand, we can look for lattice sphere packings in Euclidean $m$-space; on the other hand, we can see $T$ as a surface of genus 1 and look for systoles in surfaces of genus $g$. This shows that the problem of the densest lattice sphere packing is analogous to the problem of finding the surface with the longest systole, and the problem of finding the lattice sphere packing with best kissing number (the number of spheres 


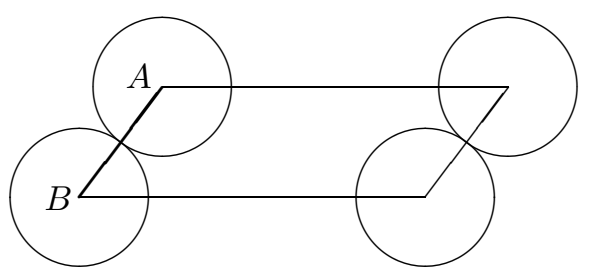

Figure 2. A lattice circle packing defined by a shortest closed geodesic of a torus

touching the same sphere) is analogous to the problem of finding the surface with the largest number of systoles. These sphere packing problems are classical in the geometry of numbers; I call the hyperbolic geometry of numbers the analogous problems for surfaces and their systoles.

The analogy described above can be expressed as an analogy between the associated groups, $S L(m, \mathrm{Z})$ on the one hand and the mapping class groups on the other hand; see Ivanov [47]. See also Bavard [6], who has put this analogy in a broader context.

Note that there is another approach to formulating the Euclidean geometry of numbers in the context of hyperbolic geometry. In it, Euclidean space is directly replaced by the hyperbolic plane (or hyperbolic space). The first problem considered in this way was a hyperbolic lattice problem, an analogue of the classical circle problem; see Delsarte [27], Huber [44]; for more recent treatments see [16] and [49]. See also Tsuji [101],[102] where the classical theorems of Minkowski and Blichfeldt are formulated for the hyperbolic plane. One can study the geometry of numbers in more general spaces; see Chalk [20] and Gruber/Lekkerkerker [38], pg. 629ff, for an overview. I emphasize however that the hyperbolic geometry of numbers as defined here is of a different nature.

I shall concentrate on the two already mentioned extremal problems. For related problems see for example [76], in particular the contribution of Quine, where he treats analogous problems in the context of Jacobians. See further Adams [2] for horocyclic packings from which he derives some results about systoles. Extremal problems have been considered in similar contexts; see in particular Bollobás [9] for extremal graphs and Ahlfors [3] for extremal problems in conformal geometry. Concerning the Euclidean geometry of numbers one may consult Minkowski [66], Siegel [96], Cassels [18], Hlawka [43], Fricker [35], Gruber/Lekkerkerker [38], Erdös/Gruber/Hammer [29], Conway/Sloane [24].

It is a surprising fact that the situation in the case of the hyperbolic geometry of numbers often looks nicer than in the classical Euclidean geometry of numbers; see for example my following conjecture.

Conjecture 1. There are universal constants $C_{1}$ and $C_{2}$ such that a surface $M \in$ $T_{g, n}$ has

(i) a systole $u$ with

$$
2 \cosh (L(u) / 2) \leq C_{1}\left(\operatorname{dim} T_{g, n}\right)^{2 / 3},
$$


(ii) at most

$$
C_{2}\left(\operatorname{dim} T_{g, n}\right)^{4 / 3}
$$

different systoles.

In the context of the Euclidean geometry of numbers one would be happy to have such nice conjectures.

What is known about Conjecture 1? Let me begin with the following result (which has no analogue in the Euclidean geometry of numbers).

Theorem 3. (Schmutz [83]) In its Teichmüller space, the surface

$$
\mathrm{H} / \Gamma(N), \quad N \geq 2,
$$

is a surface with the longest possible systole.

The dimension of the Teichmüller space of $\mathrm{H} / \Gamma(N)$ grows roughly as $N^{3}$, while the norm of a systole in $\mathrm{H} / \Gamma(N)$ is $N^{2}-2$ (if $N \geq 3$ ), which implies that the exponent in part (i) of Conjecture 1 cannot be smaller than $2 / 3$. Note also that the systoles of surfaces corresponding to the principal congruence subgroups of some co-compact arithmetic Fuchsian groups grow with a comparable size.

The proof of Theorem 3 strongly uses the fact that $\Gamma(N)$ is a normal subgroup of the modular group. That $\mathrm{H} / \Gamma(N)$ is locally extremal for the function "systole" is proved with the help of Corollary 1 in section 5 . In order to prove the global property, one applies the idea of the proof of a result of Fejes Toth [32] about best sphere packings of the hyperbolic plane; these best packings are provided by the triangle groups $(2,3, m), m \geq 7$ an integer.

Concerning part (ii) of Conjecture 1, we have the following result.

Theorem 4. (Schmutz [88]) For every $\varepsilon>0$ there are infinitely many different integers $N_{i}$ such that the surface

$$
\mathrm{H} / \Gamma\left(N_{i}\right)
$$

has more than

$$
\left(\operatorname{dim} \mathcal{T}_{i}\right)^{4 / 3-\varepsilon}
$$

different systoles $\left(\mathcal{T}_{i}\right.$ is here the Teichmüller space of $\left.\mathrm{H} / \Gamma\left(N_{i}\right)\right)$. A similar result holds for the surfaces corresponding to the principal congruence subgroups of some co-compact arithmetic Fuchsian groups.

In the case of $\Gamma(N)$ the idea of the proof is as follows. Let $\gamma$ be an element of the modular group $\Gamma(1)$ with $|\operatorname{tr}(\gamma)|=N>2$. Then, as a matter of fact, the element $-\gamma^{2}$ lies in $\Gamma(N)$ and corresponds to a systole in $\mathrm{H} / \Gamma(N)$. In order to produce many systoles in $\mathrm{H} / \Gamma(N)$ one has to choose $\gamma$ such that the modular group has many different mutually non-conjugate elements of the same trace $N=|\operatorname{tr}(\gamma)|$. By a celebrated theorem of Siegel [95] (formulated in the language of class numbers of real quadratic number fields), this multiplicity grows with $N^{1-\varepsilon}$ for every $\varepsilon>0$, and this is enough to prove Theorem 4 (instead of Siegel's theorem one may also apply Theorem 14 in section 5 below). The same idea works for some co-compact arithmetic Fuchsian groups.

In the Euclidean geometry of numbers the densest lattice sphere packing and the lattice sphere packing with the largest kissing number are known up to dimension 8; moreover, in these dimensions the extremal lattice is unique and is the same for both problems (this is certainly wrong in higher dimensions). For closed surfaces, 
the final result is only known for genus 2. In genus 3, there is a very promising candidate (compare [82]).

Theorem 5. (Jenny [50], Schmutz [82],[84]) In $T_{2}$, the (up to isometry) unique surface with an automorphism group of order 48 (the largest automorphism group for surfaces in $T_{2}$ ) is the unique global maximum for the length of the systole as well as for the number of systoles.

Conjecture 2. In $T_{3}$, the (up to isometry) unique non-hyperelliptic surface with an automorphism group of order 48 (which is not the largest automorphism group for surfaces in $T_{3}$ !) is the unique global maximum for the length of the systole as well as for the number of systoles.

Until now we have compared the lengths of the shortest closed geodesics of different surfaces of a fixed Teichmüller space. One could generalize this problem and compare the lengths of the closed geodesics which are the second shortest, and so on. Here is the precise definition.

Definition. (i) The set of lengths $\mathcal{L}(M)$ of a surface $M$ is defined as the set

$$
\mathcal{L}(M)=\left\{L_{M}(u): u \text { is a closed geodesic of } M\right\} .
$$

(ii) Let $M$ and $M^{\prime}$ be two surfaces. Let $\mathcal{L}(M)=\left\{a_{1}, a_{2}, \ldots\right\}$ and $\mathcal{L}\left(M^{\prime}\right)=$ $\left\{a_{1}^{\prime}, a_{2}^{\prime}, \ldots\right\}$ both be listed in ascending order $\left(a_{i}<a_{j}\right.$ and $a_{i}^{\prime}<a_{j}^{\prime}$ for $\left.i<j\right)$. I write

if $a_{i}^{\prime} \leq a_{i}, \forall i$.

$$
\mathcal{L}\left(M^{\prime}\right) \leq \mathcal{L}(M)
$$

(iii) $M$ has maximal lengths if and only if $\mathcal{L}\left(M^{\prime}\right) \leq \mathcal{L}(M)$ for all surfaces $M^{\prime}$ in the Teichmüller space of $M$.

Theorem 6. ([86]) The surfaces $\mathrm{H} / \Gamma(N), N>2$, have maximal lengths.

This is a generalization of Theorem 3. The proof of Theorem 6 uses the following observation. $\mathrm{H} / \Gamma(N)$ has a systole $u$ which separates the surface into two parts $Q$ and $P$ such that $P$ is a pair of pants with two cusps. By the proof of Theorem 3 it results that in every surface $M^{\prime}$ in the Teichmüller space of $\mathrm{H} / \Gamma(N)$ one can find an embedded pair of pants $P^{\prime}$ with two cusps and a boundary component $u^{\prime}$ such that $L_{M^{\prime}}\left(u^{\prime}\right) \leq L_{M}(u)$. Since the set of lengths $\mathcal{L}(P)$ equals the set of lengths $\mathcal{L}(\mathrm{H} / \Gamma(N))$, the theorem can be proved by showing $\mathcal{L}\left(P^{\prime}\right) \leq \mathcal{L}(P)$, which is not very hard.

The analogous problem had not been considered in the classical Euclidean geometry of numbers. But a somewhat weaker problem was treated in a paper of Conway/Sloane [23] where the best asymptotic growth was analysed and a solution was given for all dimensions $m>2(m=2$ seems to be open). The following theorem is part of their main theorem.

Theorem 7. ([23]) Let $\Lambda$ be a lattice in Euclidean space $\mathrm{R}^{m}$ with determinant 1 (this is the volume of a fundamental domain of $\Lambda$ ). Let

$$
E(\Lambda)=\lim _{C \rightarrow \infty} \frac{\#\{\langle x, x\rangle \leq C: x \in \Lambda\}}{C} .
$$

Let

$$
E_{0}(m)=\inf \left\{E(\Lambda): \Lambda \text { a lattice in } \mathrm{R}^{m} \text { with determinant } 1\right\} .
$$

If $3 \leq m \leq 8$, then $E_{0}(m)=E\left(\Lambda_{0}\right)$ for a unique lattice $\Lambda_{0}$, the same which realizes the best lattice sphere packing in dimension $m$. 
I conjecture that the sharper property of "maximal lengths" (it should be clear what this means here) also exists in the Euclidean geometry of numbers.

Conjecture 3. In dimensions 2 to 8 , the known best lattice sphere packings have "maximal lengths".

In addition to Theorem 7 , this conjecture is based on the fact that in these dimensions, the best lattice sphere packings are very particular (for example they also have the best kissing number).

In dimension 2, some work has been done in order to prove this conjecture for the best circle packing, the so-called hexagonal lattice; see Kühnlein [54]. In dimension 2 the conjecture means in particular that the hexagonal lattice is "better" than the square lattice. More precisely, let $0<h_{1}<h_{2}<\ldots$ be the positive integers, listed in ascending order, which can be written as

$$
h_{i}=x^{2}+3 y^{2}
$$

for integers $x$ and $y$. Let $0<q_{1}<q_{2}<\ldots$ be the positive integers, listed in ascending order, which can be written as

$$
q_{i}=x^{2}+y^{2}
$$

for integers $x$ and $y$. Then the conjecture is

$$
q_{i} \leq h_{i}, \quad \forall i=1,2,3, \ldots .
$$

Theorem 6 above gives examples of surfaces with maximal lengths. These surfaces are all non-compact. The best candidate in the compact case is the surface of genus 2 which already appeared in Theorem 5 . This surface is also known as the Bolza curve.

Conjecture 4. The Bolza curve has maximal lengths.

An often asked question is that of uniqueness. More precisely, is a surface with maximal systole unique (up to isometry) in its Teichmüller space? The answer is no, even for surfaces with maximal lengths (see [87]).

\section{Systole as a Morse function}

I denote by syst the function on $T_{g, n}$ which associates to every surface the length of a systole. This function is not differentiable since in surfaces with more than one systole, syst has a corner. While the (by syst) induced function on the moduli space of $(g, n)$-surfaces is proper (this follows by a result of Mumford [69]), syst itself is not proper. For example, if $M \in T_{g, n}$ has a systole $u$ such that all closed geodesics intersecting $u$ are "much" longer than $u$, then a twist deformation along $u$ does not change syst so that syst ${ }^{-1}\left(L_{M}(u)\right)$ will be non-compact. However, we have the following result (this section is based on [90]).

Theorem 8. (Schmutz Schaller [90])

$$
\text { syst }: T_{g, n} \longrightarrow \mathrm{R}_{+}
$$

is a topological Morse function, equivariant with respect to the mapping class group $\Gamma(g, n)$. 
The concept of a topological Morse function (introduced by Morse [68]) is not very well known. Traditionally, a Morse function is smooth. But since Morse functions are used for topological reasons, smoothness is not really needed. See for example the real valued functions

$$
f(x)=x^{3} \quad \text { and } \quad g(x)=|x| .
$$

The first function has a degenerate critical point in zero, so both functions are not Morse functions in the smooth sense. But topologically, they are equivalent to the functions

$$
\hat{f}(x)=x \quad \text { and } \quad \hat{g}(x)=x^{2} .
$$

The critical point of $f(x)$ has been removed and has become an ordinary point, while $\hat{g}(x)$ is now a smooth function with a non-degenerate critical point of index 1. Therefore, $f(x)$ and $g(x)$ are nevertheless topological Morse functions.

The definition of a non-degenerate critical point $M_{0} \in T_{g, n}$ for a continuous function $f$ (from $T_{g, n}$ into the positive reals) is the following. There exists an open neighborhood $U$ of $M_{0}$ in $T_{g, n}$, a homeomorphic parameterization of $U$ by $N=\operatorname{dim} T_{g, n}$ real parameters $y_{1}, \ldots, y_{N}$, and an integer $j$ such that

$$
f(M)-f\left(M_{0}\right)=\sum_{i=1}^{j}\left(y_{i}\right)^{2}-\sum_{i=j+1}^{N}\left(y_{i}\right)^{2}, \quad \forall M \in U .
$$

The integer $j$ is the index of the critical point. (Usually, the index is defined as the number of the negative squares, but since $\operatorname{dim} T_{g, n}$ is always even, our definition does not cause problems.)

Before giving some ideas of the proof of Theorem 8 let me make the following remarks.

There are well-known Morse functions on $T_{g, n}$. Take for example a set $F=$ $\left\{u_{1}, \ldots, u_{k}\right\}$ of $k$ simple closed geodesics which fill up $M_{0} \in T_{g, n}$ (which means that every simple closed geodesic of $M_{0}$ is intersected by an element of $F$ ). Then the function

$$
f: T_{g, n} \longrightarrow \mathrm{R}, \quad f(M)=\sum_{i=1}^{k} L_{M}\left(u_{i}\right),
$$

is a proper smooth function on $T_{g, n}$ with a unique critical point which is nondegenerate (this implies that $T_{g, n}$ is a cell). Such functions have been used for example in [52] and [108]. A function with the same properties is the energy of a harmonic map; see for example [100],[105]. The function syst on the other hand is a Morse function on $T_{g, n}$ which is equivariant with respect to $\Gamma(g, n)$. Such functions are helpful in order to study the topology of the moduli spaces (compare with Theorem 12 below), and syst seems to be the first explicit example. There are further candidates. The following conjecture is based on [71],[72].

Conjecture 5. (Sarnak $[81]$ )

$$
-\log \operatorname{det} \Delta(M)
$$

is a Morse function (this function is the (zeta regularized) product of the non-zero eigenvalues of the Laplacian of a Riemann surface $M$ ).

I guess that the function $\lambda_{1}$, which associates to every surface the value of the smallest non-zero eigenvalue of the Laplacian, is a topological Morse function. 
In the Euclidean geometry of numbers the analogue of Theorem 8 has been known for some time.

Theorem 9. (Ash [4]) The packing function on the space of (Euclidean) lattice sphere packings of dimension $m$ is a topological Morse function.

I now make some comments concerning the proof of Theorem 8.

Definition. Let $\mathcal{M} \in T_{g, n}$. Let $F$ be a set of $k$ simple closed geodesics of $\mathcal{M}$. Define the subset $\operatorname{Min}(F)$ of $T_{g, n}$ as follows. $M_{0} \in \operatorname{Min}(F)$ if and only if there exists a vector $a \in \mathrm{R}^{k}$ such that all components of $a$ are strictly positive and such that the inner product

$$
\left\langle a, L_{M}(F)\right\rangle
$$

has, if $M$ varies in $T_{g, n}$, a unique minimum in $M_{0}$. Here, $L_{M}(F)$ is the vector composed of the $k$ length functions induced by $F$.

Note that $\operatorname{Min}(F)$ is empty if the elements of $F$ do not fill up $\mathcal{M}$.

Theorem 10. ([90]) If the elements of $F$ fill up $\mathcal{M}$, then $M i n(F)$ is a differentiable submanifold of $T_{g, n}$.

For a surface $M$ denote by $S(M)$ its set of systoles. This set will take the role of $F$. The following result is slightly stronger than Theorem 8 .

Theorem 11. ([90]) $M_{0}$ is a critical point for syst iff $M_{0} \in \operatorname{Min}\left(S\left(M_{0}\right)\right) ; M_{0}$ is then non-degenerate. Moreover, up to isometry, $T_{g, n}$ has only a finite number of critical points for syst.

An element of the proof of this theorem is the following lemma which follows directly from the definitions.

Lemma 1. If $M_{0} \in \operatorname{Min}\left(S\left(M_{0}\right)\right)$, then syst restricted to $\operatorname{Min}\left(S\left(M_{0}\right)\right)$ has a unique global maximum in $M_{0}$.

By Theorem 10, this gives one half of the parameterization needed for a nondegenerate critical point. Namely, $M_{0}$ is the global maximum (of syst) with respect to the submanifold $\operatorname{Min}\left(S\left(M_{0}\right)\right)$; thus it corresponds to the sum of the negative squares.

On the other hand, let $\xi$ be a non-zero vector in the tangent space of $T_{g, n}$ in $M_{0}$. For a closed geodesic $u$ of $M_{0}$, denote by $\xi(u)$ the real number which is obtained by applying $\xi$ to the length function $L(u)$. Assume that $\xi$ is normal to $\operatorname{Min}\left(S\left(M_{0}\right)\right)$, which means that $\xi(u)=0$ for all $u \in S\left(M_{0}\right)$. Let $\gamma(\xi)$ be the Weil-Petersson geodesic (in $T_{g, n}$ ) induced by $\xi$ passing through $M_{0}$. Since the length functions are strictly convex along Weil-Petersson geodesics (Wolpert [108]), it follows that syst restricted to $\gamma(\xi)$ has an isolated local minimum in $M_{0}$. In this way, one obtains the sum of the positive squares for the desired parameterization.

Concerning the last statement of Theorem 11, observe that if $M_{1}$ and $M_{2}$ are two non-isometric critical points for syst, then $S\left(M_{1}\right)$ and $S\left(M_{2}\right)$ must be nonequivalent as topological sets (in the sense of Theorem 2 in section 3 ) since otherwise we could find a surface $N$ isometric to $M_{2}$ such that $S\left(M_{1}\right)=S(N)$ (in the sense of marked geodesics). But then a Weil-Petersson geodesic between $M_{1}$ and $N$ would imply (again since the length functions are strictly convex along Weil-Petersson geodesics) that $M_{1} \notin \operatorname{Min}\left(S\left(M_{1}\right)\right)$ or $N \notin \operatorname{Min}(S(N))$, contradicting the first part of Theorem 11. 
I further note a corollary of Theorem 11 which is an analogue to the theorem of Voronoï [104] that a positive definite quadratic form $f$ is extremal if and only if $f$ is perfect and eutactic; see also [82],[6].

Corollary 1. A $(g, n)$-surface $M$ is a local maximum for syst if and only if $M$ is a critical point of syst of index zero.

Since syst is a topological Morse function, syst gives rise to a cellular decomposition (by Morse [68]). It then follows by cohomology of groups (see in particular Brown [14]) that there is the following "mass formula" (notice the corresponding mass formula in the Euclidean geometry of numbers found by Ash [5]; see also [7]).

Theorem 12. ([90]) Let $M \in T_{g, n}$ be a critical point for syst of index $j(M)$. Let $|A u t(M)|$ be the order of the automorphism group of $M$ which contains the orientation preserving isometries which fix all cusps (if $n>0$ ). Then we have

$$
\sum_{M \in \operatorname{Crit}(g, n)} \frac{(-1)^{j(M)}}{|A u t(M)|}=\chi(\Gamma(g, n))
$$

where $\Gamma(g, n)$ is the mapping class group for $(g, n)$-surfaces with fixed cusps.

Here, $\operatorname{Crit}(g, n)$ is the set of mutually non-isomorphic critical points for syst in $T_{g, n}$ (where two critical points $M_{1}$ and $M_{2}$ are isomorphic if there exists an orientation preserving isometry $\phi$ which maps $M_{1}$ to $M_{2}$ while fixing all cusps) and $\chi(\Gamma(g, n))$ is the virtual Euler characteristic of $\Gamma(g, n)$. The virtual Euler characteristic is defined as

$$
\chi(\Gamma(g, n))=\frac{1}{J} e(T(g, n) / G)
$$

where $G$ is a torsion-free subgroup of $\Gamma(g, n)$ of finite index $J$ so that the ordinary Euler characteristic $e(T(g, n) / G)$ of $T(g, n) / G$ is well defined (the rational number $\chi(\Gamma(g, n))$ does not depend on $G)$.

The number $\chi(\Gamma(g, n))$ has been calculated independently by Harer/Zagier [41] and Penner [73]; see also Harer [40].

In low-dimensional cases, it is possible to determine the set $\operatorname{Crit}(g, n)$ of (isomorphism classes of) critical points for syst. For example, there are exactly four (mutually non-isometric) critical points for closed surfaces of genus 2; see [90].

Problem. Determine the critical points of syst for closed surfaces of genus 3 .

\section{Geometric Characterization of ARithmetic}

FUCHSIAN GROUPS AND THE MULTIPLICITY IN THE LENGTH SPECTRUM

Arithmetic Fuchsian groups are the most important and most interesting Fuchsian groups. This is also due to their geometric properties. A striking example is the fact that the modular group is the non-compact Fuchsian group with the smallest area (of a corresponding fundamental domain). In the geometric approach to Riemann surface theory based on closed geodesics, arithmetic Fuchsian groups appear in a natural way. These groups are often critical points for the function syst despite the fact that in every Teichmüller space there exists, up to isometry, only a finite number of surfaces which correspond to arithmetic Fuchsian groups. For example, it was mentioned above that there exist exactly four (mutually nonisometric) critical points for syst in $T_{2}$, and it turns out that all of them correspond 
to arithmetic Fuchsian groups; see [90]. It is therefore natural to ask for a geometric characterization of arithmetic Fuchsian groups.

Definition. (i) The trace set of a Fuchsian group $\Gamma$ is the set

$$
\operatorname{Tr}(\Gamma)=\{|\operatorname{tr}(u)|: u \text { is a hyperbolic element in } \Gamma\} .
$$

(ii) Let $\Gamma$ be a Fuchsian group. Define the subgroup $\Gamma^{2}$ as

$$
\Gamma^{2}=\left\langle\gamma^{2}: \gamma \in \Gamma\right\rangle \text {. }
$$

(iii) An arithmetic Fuchsian group is a co-finite Fuchsian group $\Gamma$ with the following two properties.

a) $K=\mathrm{Q}[\operatorname{Tr}(\Gamma)]$ is a number field of finite degree $k=[K: \mathrm{Q}]$ and $\operatorname{Tr}(\Gamma)$ is contained in the ring of integers of $K$.

b) Let $\phi$ be any of the $k$ embeddings of $K$ into the complex numbers such that $\phi$ restricted to $\operatorname{Tr}\left(\Gamma^{2}\right)$ is not the identity. Then $\phi\left(\operatorname{Tr}\left(\Gamma^{2}\right)\right) \subset[-2,2]$.

(iv) An arithmetic Fuchsian group $\Gamma$ is derived from a quaternion algebra if $\phi(\operatorname{Tr}(\Gamma))$ $\subset[-2,2]$ for every embedding $\phi$ (of $K=\mathrm{Q}[\operatorname{Tr}(\Gamma)]$ into the complex numbers) which is not the identity.

Remark. Arithmetic Fuchsian groups were originally defined as the images in $P S L(2, \mathrm{R})$ of unit groups of orders of quaternion algebras over totally real number fields; see for example [97],[51] for a precise definition. Takeuchi [97] found the above (equivalent) characterization of arithmetic Fuchsian groups which I use as a definition (see also [42]).

Definition. Let $\Gamma$ by a Fuchsian group. Define

(i) $\operatorname{Gap}(\Gamma)=\inf \{|a-b|: a, b \in \operatorname{Tr}(\Gamma), a \neq b\}$.

(ii) $\operatorname{Tr}(\Gamma)$ has linear growth if there exists a constant $C$ such that

$$
\text { \# }\{a \in \operatorname{Tr}(\Gamma): a<x\}<C x, \forall x>2 .
$$

Theorem 13. (Schmutz [86]) Let $\Gamma$ be a co-finite, not co-compact, Fuchsian group. Then

(i) $\Gamma$ is arithmetic if and only if $\operatorname{Tr}(\Gamma)$ has linear growth.

(ii) $\Gamma$ is derived from a quaternion algebra if and only if $\operatorname{Gap}(\Gamma)>0$.

Conjecture 6. The theorem also holds in the co-compact case.

The proof of the theorem relies on the analysis of the trace set of two-generator subgroups of $P S L(2, \mathrm{R})$, given in the following lemma. (In the lemma, the group $G(x)$ is not necessarily discrete, but $\operatorname{Tr}(G(x))$ is nevertheless well defined.)

Lemma 2. Let $x \in \mathrm{R}$. Let $G(x)$ be the subgroup of $P S L(2, \mathrm{R})$ generated by $T$ and $R$ with

$$
T=\left[\begin{array}{ll}
1 & 1 \\
0 & 1
\end{array}\right], \quad R=\left[\begin{array}{ll}
1 & 0 \\
x & 1
\end{array}\right] .
$$

Then $\operatorname{Tr}(G(x))$ has linear growth if and only if $x \in \mathrm{Z}$.

Now if $\Gamma$ is a co-finite, but not co-compact, Fuchsian group, then $\Gamma$ contains a parabolic element so that we may assume that $\Gamma$ contains the element $T$ (of Lemma 2 ) since the statement of Theorem 13 does not depend on conjugation in $\operatorname{PSL}(2, \mathrm{R})$. Let

$$
\gamma=\left[\begin{array}{ll}
a & b \\
c & d
\end{array}\right] \in \Gamma, \quad c \neq 0
$$


Let $Q$ be the subgroup of $\Gamma$ generated by $T$ and $\gamma T \gamma^{-1}$. Then $\operatorname{Tr}(Q)=\operatorname{Tr}(G(x))$ with $x=c^{2}$ where $G(x)$ is defined as in Lemma 2. Theorem 13 then follows rather easily from Lemma 2. Concerning the proof of Lemma 2, observe that ( $T$ and $R$ are defined as in Lemma 2)

$$
\left\{\operatorname{tr}\left(T^{m} R T^{n} R\right): m, n \in \mathbf{Z}, m>n>0\right\}=\left\{m n x^{2}+2(m+n) x+2: m>n>0\right\}
$$

and, if $x$ is not rational, then all numbers in this set are different. It follows that the order of this set grows, with respect to $N=m n$, at least as $N \log N$, hence faster than linear and Lemma 2 follows. If $x$ is rational, then the proof of Lemma 2 is a bit more difficult.

It might be possible to prove Conjecture 6 by a similar method as Theorem 13 . The problem is to find some analogue to Lemma 2 (in the compact case, we do not have the very convenient element $T$ ).

I now turn to the question of comparing the growth of the set of lengths of a surface with that of the whole length spectrum (note that the length spectrum is not a set; see the following definition).

Definition. (i) The length spectrum of a surface $M$ is the list of lengths of closed geodesics $u_{i}$ of $M$ in ascending order

$$
L\left(u_{1}\right) \leq L\left(u_{2}\right) \leq L\left(u_{3}\right) \ldots .
$$

(ii) The multiplicity of a closed geodesic $u$ in a surface $M$ is the number of closed geodesics in $M$ which have the same length as $u$.

(iii) A prime geodesic in a surface $M$ is a closed geodesic traversed once.

One of the first results concerning closed geodesics was the formula for the growth of the length spectrum. Usually, for this growth, one considers only prime geodesics. Due to the analogy with the prime number theorem the following theorem is often called the prime geodesic theorem; it is usually attributed to Selberg, even if there is no written proof by him. Independently, Huber [45] gave a proof for closed surfaces with a slightly different error term. Proofs of Theorem 14 appeared in [80] and [103]. In the theorem the numbers $s_{k}$ are defined as follows. If $0<\lambda_{k}<1 / 4$ is an eigenvalue of the Laplacian on $M$, then set

$$
s_{k}=1 / 2+\sqrt{1 / 4-\lambda_{k}} .
$$

Theorem 14. Let $M$ be a surface of finite area. Let $\pi(x)$ be the number of prime geodesics in $M$ with norm smaller than $x$. Then

$$
\pi(x)=l i\left(x^{2}\right)+\sum_{3 / 4>s_{k}>1 / 2} l i\left(x^{2 s_{k}}\right)+O\left(x^{3 / 2} / \log x\right) .
$$

In the literature, the prime geodesic theorem is usually given with half of the norm of the prime geodesics; as a consequence all exponents in the theorem are reduced to half and the analogy to the prime number theorem becomes obvious. A major question is the improvement of the error term. In analogy to the Riemann hypothesis one expects that the error term should be $O\left(x^{1+\epsilon}\right)$ for every positive $\epsilon$ (note again that the exponent is the double of the usual one), but the unique improvement found so far is for the modular group where $O\left(x^{1.4+\epsilon}\right)$ has been shown (Luo/Sarnak [58]), improving a result of Iwaniec [48]. The question of improvement of the error term is however not a geometric question, and I shall not treat it here. 
The question in which I am interested here is that of the growth of the set of lengths or, to put it a different way, the size of the multiplicity in the length spectrum. This question has attracted some interest in recent years since it is related to questions in quantum chaos; compare Luo/Sarnak [57], Ginzburg/Rudnick [36], Marklof [60]. The following conjecture expresses the general thinking.

Conjecture 7. For a generic surface $M$ of finite area, the set of norms

$$
\{2 \cosh (L(u) / 2): u \text { is a closed geodesic of } M\}
$$

grows "quadratically" (meaning that the main term is not different from the growth in the prime geodesic theorem).

It is quite clear that the method of analysing pairs of pants as in Lemma 2 (note that a pair of pants has a corresponding two-generator Fuchsian group) is not powerful enough for such a result. Namely, it follows by the prime geodesic theorem and a result of Guillopé [39] that the length spectrum (taking the norms as in Theorem 14) of a generic pair of pants has a growth which is only linear times logarithmic terms.

One may express this fact in a slightly different way. The proof of Lemma 2 uses the difference in the growth of the trace sets of generic two-generator Fuchsian groups and of "arithmetically defined" two-generator Fuchsian groups (the Fuchsian groups $G(x), x>4$, in Lemma 2 are not co-finite, therefore they are not arithmetic). This difference is very small, namely linear versus linear times logarithmic terms. But for Fuchsian groups of finite volume the expected difference is much bigger, namely linear versus quadratic.

A very interesting question is the following. It is known [57] that the trace set of arithmetic Fuchsian groups has linear growth. By Conjecture 7 one expects (and the arguments are convincing) that the trace set of generic co-finite Fuchsian groups has quadratic growth. But is there anything between these extremal cases? At this point, no real argument has been found to conjecture reasonably whether the answer to this question is "yes" or "no". If the answer is "yes", it would be very interesting to determine the class of Fuchsian groups which have intermediate growth (between linear and quadratic). A natural candidate for such a group is the triangle group $(2,5, \infty)$ which is the "simplest" non-arithmetic Fuchsian group.

However, there is an argument making it plausible that the growth of the trace set of non-arithmetic co-finite Fuchsian groups is not close to linear. Namely, by Margulis (see [59], [109] and the references therein) arithmetic Fuchsian groups are characterized by the fact that their commensurator is not a Fuchsian group; the commensurator of a Fuchsian group $\Gamma$ is the group

$$
\left\{\gamma \in P S L(2, \mathrm{R}): \Gamma \text { and } \gamma \Gamma \gamma^{-1} \text { are commensurable }\right\} .
$$

By a result of Borel [12], in the commensurability class of an arithmetic Fuchsian group, there are infinitely many mutually non-conjugate maximal Fuchsian groups (here, a Fuchsian group $\Gamma$ is maximal if $\Gamma$ is not contained in a Fuchsian group different from $\Gamma$ ). On the other hand, by the result of Margulis, in the commensurability class of a co-finite non-arithmetic Fuchsian group, there is a unique maximal Fuchsian group. This difference should imply that the multiplicity is much higher in the length spectrum of surfaces corresponding to arithmetic Fuchsian groups.

In order to better understand the multiplicity of the length spectrum one also has to understand the so-called stable multiplicity. This multiplicity is produced 
by trace identities and does not change if one changes the hyperbolic metric of the surface. Based on this stable multiplicity and a construction of Horowitz, the following important theorem was derived.

Theorem 15. (Randol [77]) In every $(g, n)$-surface the multiplicities in the length spectrum are unbounded.

The stable multiplicity relies on the fact that every co-finite Fuchsian group has (infinitely many) two-generator subgroups such that their corresponding surfaces have non-trivial automorphism group. It is expected that stable multiplicity can only be produced by these two-generator subgroups, which would in some sense agree with the general principle that "multiplicity is produced by symmetry". More precisely, I conjecture the following. (The automorphism group of a Fuchsian group is here the automorphism group of the corresponding surface.)

Conjecture 8. Every subgroup $\Gamma^{\prime}$ of a generic co-finite Fuchsian group $\Gamma$ with trivial automorphism group has trivial automorphism group with the obvious exceptions which are:

(i) $\Gamma^{\prime}$ is cyclic or a two-generator group.

(ii) $\Gamma^{\prime}$ is a normal subgroup of a subgroup of $\Gamma$.

A part of the conjecture is proved by the following argument. If $\Gamma$ has trivial automorphism group and equals its commensurator, then every subgroup $\Gamma^{\prime} \subset \Gamma$ of finite index has trivial automorphism group (if $\Gamma^{\prime}$ is not a normal subgroup of a subgroup of $\Gamma$ ).

\section{Simple Closed Geodesics of the OnCE-PUnCtured torus}

AND THE MARKOFF SPECTRUM

The once-punctured torus (this is a $(1,1)$-surface) is a particularly interesting example where geometry (based on simple closed geodesics) and number theory join.

The Markoff spectrum [61] is defined as follows (for general information see Cassels [18], Cusick/Flahive [25], Pollington/Moran [75]). For a real number $\theta$ and (rational) integers $p, q$ let

$$
\nu(\theta)=\inf \left\{c:|\theta-p / q|<c / q^{2} \text { for infinitely many } q\right\} .
$$

Then there exists a discrete set of values $\nu_{i}$, the Markoff spectrum, decreasing to $1 / 3$, with $1 / 3$ as unique cluster point, such that if $\nu(\theta)>1 / 3$, then $\nu(\theta)$ is equal to one of the $\nu_{i}$.

An equivalent definition is that of the integer solutions of the Diophantine equation

$$
X^{2}+Y^{2}+Z^{2}=3 X Y Z
$$

A Markoff triple is a solution $(x, y, z)$ of this equation with $0<x \leq y \leq z$, and $x, y, z$ are called Markoff numbers. It was first observed by Cohn [22] that they can be interpreted geometrically; see also Gorskov [37]. Later on, Lehner/Sheingorn [55] remarked that the Markoff numbers correspond to simple closed geodesics in the modular torus $M^{\prime}$, which is the unique once-punctured torus which is produced by a subgroup of the modular group. As a matter of fact this surface has the longest systole and even has maximal lengths; see [87].

The so-called uniqueness conjecture states that a Markoff triple $(x, y, z)$ is determined by $z$. In geometric language this means that if $u$ and $v$ are simple closed 
geodesics of the same length in the modular torus $M^{\prime}$, then $M^{\prime}$ has an isometry $\phi$ such that $\phi(u)=v$. As a corollary it would follow that, in $M^{\prime}$, the maximal number of different simple closed geodesics of the same length is 6 ; more precisely, for every simple closed geodesic the multiplicity is either 3 or 6 (the multiplicity is here the number of different simple closed geodesics of the same length). Therefore, we have a geometric generalization of the uniqueness conjecture.

Conjecture 9. Let $M$ be a once-punctured torus. Let $u_{1}, \ldots, u_{k}$ be simple closed geodesics of $M$ of the same length. Then $k \leq 6$.

This is a special case of a more general conjecture (which contrasts with Theorem 15 in section 6).

Conjecture 10. (Rivin [79]) For every $(g, n)$-surface $M$ there is a constant $k(g, n)$, depending only on $g$ and $n$, such that if $u_{1}, \ldots, u_{k}$ are simple closed geodesics in $M$ of the same length, then $k \leq k(g, n)$.

But until now, no one has been able to prove for a single $(g, n)$-surface $M$, $(g, n) \neq(0,3)$, that the number of different simple closed geodesics of the same length is bounded!

The once-punctured torus has other interesting properties. For example there is the following striking result.

Theorem 16. (McShane [62],[63]) Let $M$ be a once-punctured torus. Then

$$
\sum_{u} \frac{1}{1+\exp \left(L_{M}(u)\right)}=\frac{1}{2}
$$

where the sum is over all simple closed geodesics $u$ of $M$.

Concerning the proof observe first that every simple closed geodesic $u$ of a oncepunctured torus $M$ defines a unique simple geodesic $c(u)$ which starts and ends in the cusp of $M$ and does not intersect $u$. Let $H$ be a small horocycle of the cusp in $M$, and let $E$ be the subset of $H$ containing a point $x$ if and only if $M$ has a complete simple geodesic which passes through $x$. Theorem 16 is proved by a detailed analysis of the set $E$ (for example, $c(u) \cap E$ is an isolated point in $E$ for every simple closed geodesic $u$ of $M$ ) together with the application of a result of Birman/Series [8].

An alternative proof of Theorem 16 using Markoff triples has been given by Bowditch [13].

The set of lengths of simple closed geodesics of the once-punctured torus has also been studied by McShane/Rivin [64],[65], who give in particular an estimation for the growth of this set.

\section{Small eigenvalues of the Laplacian}

Let $M \in T_{g}$. Then $M$ has only a bounded number of Laplacian eigenvalues smaller than $1 / 4$; these eigenvalues will be called small eigenvalues (note that I call the eigenvalue zero also a small eigenvalue). What is their maximal number? This is a geometric problem; there are convincing arguments in favour of the conjecture that there are at most $2 g-2$ small eigenvalues.

For an estimation of the first non-zero small eigenvalue $\lambda_{1}(M)$ of $M$, there is a direct relation to closed geodesics through Cheeger's inequality, which states the following (for the material of this section I refer the reader to [85] and [16]). 
Theorem 17. (Cheeger's inequality) Put

$$
h(M)=\inf \frac{L(A)}{\min \left\{\operatorname{area}(B), \text { area }\left(B^{\prime}\right)\right\}}
$$

where $A$ ranges over the set of piecewise smooth curves in $M$ which separate $M$ into two disjoint connected relatively open subsets $B$ and $B^{\prime}$, and $L(A)$ is the length of A. Then

$$
\lambda_{1}(M) \geq \frac{1}{4} h^{2}(M) .
$$

It follows that for $\lambda_{1}<1 / 4$, Cheeger's inequality provides an upper bound for the length of the systole of $M$. For relations of small eigenvalues with estimations of lengths of short closed geodesics see also [15],[92],[21],[28].

In this section I describe a further connection between the maximal number of small eigenvalues and estimations of lengths of closed geodesics.

Buser [15] showed that a closed surface of genus $g$ has at most $4 g-2$ small eigenvalues. Later, he also proved the fact that there is a universal $\varepsilon>0$ such that a closed surface of genus $g$ has at most $2 g-2$ eigenvalues smaller than $\varepsilon$ (see [16]); this is an important result since the bound $\varepsilon$ is independent of $g$.

The upper bound for the number of small eigenvalues was improved in Schmutz [85] to $4 g-5$. The proof of this result uses the following fact.

Theorem 18. ([85]) Every closed surface has an embedded pair of pants which has no non-zero small eigenvalue with respect to Neumann boundary conditions.

Again, the importance of the theorem relies on the fact that it is independent of $g$. The theorem can be reformulated in terms of simple closed geodesics.

Theorem 19. Every closed surface has an embedded "good" pair of pants where a good pair of pants is one which satisfies (at least) one of the following two conditions (the lengths of the boundary geodesics of the pair of pants being $2 x, 2 y, 2 z$ with $x \leq y \leq z)$.

(i) $\cosh z<9$

$$
\frac{1}{5} \sinh z<\sinh (x+y)<5 \sinh z .
$$

Notice that the bounds $9,1 / 5$ and 5 are not sharp.

A partition of a surface $M \in T_{g}$ is a set of $3 g-3$ mutually disjoint simple closed geodesics of $M$. A partition separates $M$ into $2 g-2$ pairs of pants with mutually disjoint interior. Theorem 19 gave me the idea of the following conjecture which, if true, seems to be a very strong result (which, for example, would have as a corollary that the maximal number of small eigenvalues is $2 g-2$ ).

Conjecture 11. Every closed surface has a partition which separates the surface into $2 g-2$ good pairs of pants.

The conjecture has been proved (see [85]) for $g=2$. But this result is not a really convincing argument for the truth of Conjecture 11, since in the case $g=2$ it is sufficient to use the first condition $\cosh z<9$ in Theorem 19. However, if $g$ is big enough (this might be the case for $g \geq 3$ already), then the second condition

$$
\frac{1}{5} \sinh z<\sinh (x+y)<5 \sinh z
$$

becomes the crucial one. 
An interesting problem may also be the following. For a fixed closed surface $M$, what is the statistical frequency of good pairs of pants among all pairs of pants embedded in $M$ ?

\section{REFERENCES}

[1] W. Abikoff. The real analytic theory of Teichmüller space. Springer LNM 820 (1980). MR 82a: 32028

[2] C. Adams. Maximal cusps, collars and systoles in hyperbolic surfaces. To appear in Indiana Univ. Math. J.

[3] L.V. Ahlfors. Conformal invariants. Mc Graw-Hill (1973). MR 50:10211

[4] A. Ash. On eutactic forms. Can. J. Math. 29 (1977), 1040-1054. MR 58:10762

[5] A. Ash. On the existence of eutactic forms. Bull. London Math. Soc. 12 (1980), 192-196. MR 83a: 10034

[6] C. Bavard. Systole et invariant d'Hermite. J. reine angew. Math. 482 (1997), 93-120. MR 98a:11085

[7] A.M. Bergé; J. Martinet. Sur la classification des réseaux eutactiques. J. London Math. Soc. 53 (1996), 417-432. MR 97i: 11076

[8] J.S. Birman; C. Series. Geodesics with bounded intersection number on surfaces are sparsely distributed. Topology 24 (1985), 217-225. MR 87f:57012

[9] B. Bollobás. Extremal graph theory. Academic Press (1978). MR 80a:05120

[10] F. Bonahon. The geometry of Teichmüller spaces via geodesic currents. Inv. math. 92 (1988),139-162. MR 90a:32025

[11] F. Bonahon. Shearing hyperbolic surfaces, bended pleated surfaces and Thurston's symplectic form. Ann. Fac. Sci. Toulouse (1996), 233-297. MR 97i:57011

[12] A. Borel. Commensurability classes and volumes of hyperbolic 3-manifolds. Ann. Scuola Norm. Sup. Pisa (Ser. IV) 8 (1981), 1-33. MR 82j:22008

[13] B.H. Bowditch. A proof of McShane's identity via Markoff triples. Bull. London Math. Soc. 28 (1996), 73-78. MR 96i:58137

[14] K.S. Brown. Cohomology of groups. Springer (1982). MR 96a:20072

[15] P. Buser. Riemannsche Flächen mit Eigenwerten in $(0,1 / 4)$. Comment. Math. Helv. 52 (1977), 25-34. MR 55:7924

[16] P. Buser. Geometry and spectra of compact Riemann surfaces. Birkhäuser (1992). MR 93g:58149

[17] R.D. Canary; D.B.A. Epstein; P. Green. Notes on notes of Thurston. In Analytical and geometrical aspects of hyperbolic space. D.B.A. Epstein ed. Cambridge Univ. Press (1987), 3-92. MR 89e:57008

[18] J.W.S. Cassels. An introduction to the geometry of numbers. Second ed. Springer (1971). MR 46:5257

[19] A.J. Casson; S.A. Bleiler. Automorphisms of surfaces after Nielsen and Thurston. Cambridge University Press (1988). MR 89k:57025

[20] J.H.H. Chalk. Algebraic lattices. In Convexity and its applications. P.Mp. Gruber; J.M. Wills eds. Birkhäuser (1983), 97-110. MR 85c:11054

[21] I. Chavel. Eigenvalues in Riemannian geometry. Academic Press (1984). MR 86g:58140

[22] H. Cohn. Approach to Markoff's minimal forms through modular functions. Annals Math. 61 (1955), 1-12. MR 16:801e

[23] J.H. Conway; N.J.A.Sloane. Lattices with few distances. J. Number theory 39 (1991), 75-90.

[24] J.H. Conway; N.J.A.Sloane. Sphere packing, lattices and groups. Second ed. Springer (1993). MR 93h:11069

[25] T. Cusick; M. Flahive. The Markoff and Lagrange Spectra. Math. Surveys, vol. 30, AMS (1989). MR 90i:11069

[26] M. Dehn. Papers on group theory and topology. J. Stillwell ed. Springer (1987). MR 88d:01041

[27] J. Delsarte. Sur le gitter fuchsien. C.R. Acad. Sci. Paris Sér. I Math. 214 (1942), 147-149. MR 4:191

[28] J. Dodziuk; T. Pignataro; B. Randol; D. Sullivan. Estimating small eigenvalues of Riemann surfaces. In The legacy of Sonya Kovalevskaya. Contemp. Math 64, AMS (1987), 93-121. MR 88h:58119 
[29] P. Erdös; P.M. Gruber; J. Hammer. Lattice points. Longman Scientific \& Technical Essex (1989). MR 90g:11081

[30] H.M. Farkas; I. Kra. Riemann surfaces. 2nd ed. Springer (1992). MR 93a:30047

[31] A. Fathi; F. Laudenbach; V. Poénaru. Travaux de Thurston sur les surfaces. Séminaire Orsay, Astérisque 66-67 (1979).

[32] L. Fejes Tóth. Kreisausfüllungen der hyperbolischen Ebene. Acta Math. Acad. Sci. Hungar 4 (1953), 103-110.

[33] W. Fenchel; J. Nielsen. Discontinuous groups of non-euclidean motions. Unpublished manuscript (1948).

[34] R. Fricke; F. Klein. Vorlesungen über die Theorie der elliptischen Modulfunktionen. Band 1,2. Teubner Leipzig $(1890,1892)$.

[35] F. Fricker. Lehrbuch der Gitterpunktlehre. Birkhäuser (1982).

[36] D. Ginzburg; Z. Rudnick. Stable multiplicities in the length spectrum of Riemann surfaces. To appear in Israel J. Math.

[37] D.D. Gorskov. Geometry of Lobachevski in connection with certain questions in arithmetic. J. Soviet Math. 16 (1981), 780-820.

[38] P.M. Gruber; C.G. Lekkerkerker. The geometry of numbers. Second ed. North-Holland (1987). MR 88j:11034

[39] L. Guillopé. Sur la distribution des longueurs des géodésiques fermées d'une surface compacte à bord totalement géodésique. Duke Math. J. 53 (1986), 827-848. MR 88e:11042

[40] J.L. Harer. The cohomology of moduli space of curves. In Theory of moduli. Sernesi ed. Springer LNM 1337 (1988), 138-221. MR 90a:32026

[41] J.L. Harer; D. Zagier. The Euler characteristic of the moduli space of curves. Invent. math. 85 (1986), 457-485. MR 87i:32031

[42] H.M. Hilden; M.T. Lozano; J.M. Montesinos-Amilibia. A characterization of arithmetic subgroups of $S L(2, \mathrm{R})$ and $S L(2, \mathrm{C})$. Math. Nachr. 159 (1992), 245-270. MR 94i:20088

[43] E. Hlawka. 90 Jahre Geometrie der Zahlen. Jahresbuch Ueberblick Mathematik (1980), 9-41. MR 83a: 10051

[44] H. Huber. Ueber eine neue Klasse automorpher Formen und ein Gitterproblem in der hyperbolischen Ebene I. Comment. Math. Helv. 30 (1955), 20-62.

[45] H. Huber. Zur analytischen Theorie hyperbolischer Raumformen und Bewegungsgruppen. I, Math. Ann. 138 (1959), 1-26; II, Math. Ann. 142 (1961), 385-398. MR 22:99; MR 23:A3845

[46] Y. Imayoshi; M. Taniguchi. An introduction to Teichmüller spaces. Springer (1992). MR 94b:32031

[47] N.V. Ivanov. Complexes of curves and the Teichmüller modular group. Uspekhi Mat. Nauk 42 (1987), 43-91; English transl. Russian Math. Surveys 42 (1987), 55-107. MR 88g:32040

[48] H. Iwaniec. Prime geodesic theorem. J. reine angew. Math. 349 (1984), 136-159. MR 85h: 11025

[49] H. Iwaniec. Introduction to the spectral theory of automorphic forms. Revista Matemática Iberoamericana (1995). MR 96f: 11078

[50] F.W. Jenny. Ueber den ersten Eigenwert des Laplace-Operators auf einer Schar kompakter Riemannscher Flächen. Comment. Math. Helv. 59 (1984), 193-203.

[51] S. Katok. Fuchsian groups. The University of Chicago Press (1992). MR 93d:20088

[52] S.P. Kerckhoff. The Nielsen realization problem. Annals Math. 117 (1983), 235-265. MR 85e:32029

[53] S.P. Kerckhoff. Lines of minima in Teichmüller space. Duke Math. J. 65 (1992), 187-213. MR 93b:32027

[54] S. Kühnlein. Partial solution of a conjecture of Schmutz. Archiv d. Math. 67 (1996), 164-172. MR 97h:11069

[55] J. Lehner; M. Sheingorn. Simple closed geodesics on $\Gamma(3)$ arise from the Markoff spectrum. Bulletin AMS 11 (1984), 359-362. MR 86b:11033

[56] F. Luo. Geodesic length functions and Teichmüller spaces. To appear in J. Diff. Geom. MR 98a:32023

[57] W. Luo; P. Sarnak. Number variance for arithmetic hyperbolic surfaces. Commun. Math. Phys. 161 (1994), 419-432. MR 95k:11076

[58] W. Luo; P. Sarnak. Quantum ergodicity of eigenfunctions on $P S L_{2}(\mathbf{Z}) \backslash \mathbf{H}^{2}$. IHES Publ. Math. 81 (1995), 207-237. MR 97f:11037

[59] G.A. Margulis. Discrete subgroups of semisimple Lie groups. Springer (1991). MR 92h:22021 
[60] J. Marklof. On multiplicities in length spectra of arithmetic hyperbolic three-orbifolds. Nonlinearity 9 (1996), 517-536. MR 97d:11086

[61] A. Markoff. Sur les formes binaires indéfinies. Math. Annalen 15 (1879), 381-406.

[62] G. McShane. A remarkable identity for lengths of curves. PhD Thesis, University of Warwick (1991).

[63] G. McShane. Simple geodesics and a series constant over Teichmüller space. To appear in Invent. math.

[64] G. McShane; I. Rivin. Simple curves on hyperbolic tori. C. R. Acad. Sci. Paris 320 (1995), 1523-1528. MR 96g:57018

[65] G. McShane; I. Rivin. A norm on homology of surfaces and counting simple geodesics. Internat. Math. Res. Notices (1995), 61-69. MR 96b:57014

[66] H. Minkowski. Geometrie der Zahlen. Teubner 1896/1910; Chelsea (1953).

[67] R. Miranda. Algebraic curves and Riemann surfaces. Graduate studies in math. vol. 5, AMS (1995). MR 96f: 14029

[68] M. Morse. Topologically non-degenerate functions on a compact n-manifold M. J. Analyse Math. 7 (1959), 189-208. MR 22:4071

[69] D. Mumford. A remark on Mahler's compactness theorem. Proc. AMS 28 (1971), 289-294. MR 43:2157

[70] J. Nielsen. Collected mathematical papers. Vols. 1 and 2. Birkhäuser (1986). MR 88a:01070a; MR 88a:01070b

[71] B. Osgood; R. Phillips; P. Sarnak. Extremals of determinants of Laplacians. J. Funct. Anal. 80 (1988), 148-211. MR 90d:58159

[72] B. Osgood; R. Phillips; P. Sarnak. Moduli space, heights and isospectral sets of plane domains. Ann. Math. 129 (1989), 293-362. MR 91a:58196

[73] R.C. Penner. Perturbative series and the moduli space of Riemann surfaces. J. Diff. Geom. 27 (1988), 35-53. MR 89h:32045

[74] R.C. Penner with J.L. Harer. Combinatorics of train tracks. Princeton University Press (1992). MR 94b:57018

[75] A. Pollington; W. Moran. Number theory with an emphasis on the Markoff spectrum. Marcel Dekker (1993). MR 93m:11002

[76] J. Quine; P. Sarnak eds. Extremal Riemann surfaces. Contemp. Math. 201, AMS (1997). MR 97g:00020

[77] B. Randol. The length spectrum of a Riemann surface is always of unbounded multiplicity. Proc. AMS 78 (1980), 455-456. MR 80k:58100

[78] B. Riemann. Grundlagen für eine allgemeine Theorie der Functionen einer veränderlichen complexen Grösse. In Gesammelte Werke. Collected papers. Springer + Teubner, Leipzig (1990), 35-75.

[79] I. Rivin. Private communication.

[80] P. Sarnak. Prime geodesic theorems. Doctoral thesis, Stanford (1980).

[81] P. Sarnak. Extremal geometries. In [76], 1-7. MR 98a:58043

[82] P. Schmutz. Riemann surfaces with shortest geodesic of maximal length. Geom. Funct. Anal. 3 (1993), 564-631. MR 95j:30039

[83] P. Schmutz. Congruence subgroups and maximal Riemann surfaces. J. Geom. Anal. 4 (1994), 207-218. MR 95i:57017

[84] P. Schmutz. Systoles of Riemann surfaces. Manuscripta Math. 85 (1994), 429-447. MR 95i: 57017

[85] P. Schmutz. New results concerning the number of small eigenvalues on Riemann surfaces. J. reine angew. Math. 471 (1996), 201-220. MR 97e:58225

[86] P. Schmutz. Arithmetic groups and the length spectrum of Riemann surfaces. Duke Math. J. 84 (1996), 199-215. MR 98a:11068

[87] P. Schmutz Schaller. The modular torus has maximal length spectrum. Geom. Funct. Anal. 6 (1996), 1057-1073. MR 98c:11046

[88] P. Schmutz Schaller. Extremal Riemann surfaces with a large number of systoles. In [76], 9-19. MR 98c: 11064

[89] P. Schmutz Schaller. Geometric characterization of hyperelliptic Riemann surfaces. Preprint (1996).

[90] P. Schmutz Schaller. Systole is a topological Morse function for Riemann surfaces. Preprint (1997). 
[91] P. Schmutz Schaller. Teichmüller space and fundamental domains of Fuchsian groups. Preprint (1997).

[92] R. Schoen; S. Wolpert; S.-T. Yau. Geometric bounds on the low eigenvalues of a compact surface. In: Geometry of the Laplace operator. Proc. Symp. Pure Math. AMS 36 (1980), 279-285. MR 81i:58052

[93] A. Selberg. Harmonic analysis and discontinuous groups in weakly symmetric Riemannian spaces with applications to Dirichlet series. J. Indian Math. Soc. 20 (1956), 47-87. MR 19:531g

[94] M. Seppälä; T. Sorvali. Geometry of Riemann surfaces and Teichmüller spaces. NorthHolland (1992). MR 94b:32034

[95] C.L. Siegel. Ueber die Klassenzahl quadratischer Zahlkörper. Acta Arith. 1 (1935), 83-86.

[96] C.L. Siegel. Lectures on the geometry of numbers. Springer (1989). MR 91d:11070

[97] K. Takeuchi. A characterization of arithmetic Fuchsian groups. J. Math. Soc. Japan 27 (1975), 600-612. MR 53:2842

[98] W.P. Thurston. Earthquakes in two-dimensional hyperbolic geometry. In Low-dimensional topology and Kleinian groups, D.B.A. Epstein ed. Cambridge Univ. Press (1986), 91-112. MR 88m:57015

[99] W.P. Thurston. On the geometry and dynamics of diffeomorphisms of surfaces. Bull. AMS 19 (1988), 417-431. MR 89k:57023

[100] A.J. Tromba. Teichmüller theory in Riemannian geometry. Birkhäuser (1992). MR 93h:32028

[101] M. Tsuji. Theorems in the geometry of numbers for Fuchsian groups. J. Math. Soc. Japan 4 (1952), 189-193. MR 14:623f

[102] M. Tsuji. Analogue of Blichfeldt's theorem for Fuchsian groups. Comment. Math. Univ. St. Paul 5 (1956), 7-24. MR 18:383a

[103] A.B. Venkov. Spectral theory of automorphic functions. Trudy Mat. Inst. Steklov 153 (1981), 3-171 (Russian); English transl. in Proc. Steklov Inst. Math. (1982), issue 4, 1-163. MR 85j: $11060 b$

[104] G. Voronoï. Sur quelques propriétés des formes quadratiques positives parfaites. J. reine angew. Math. 133 (1908), 97-178.

[105] M. Wolf. The Teichmüller theory of harmonic maps. J. Diff. Geom. 29 (1989), 449-479. MR 90h: 58023

[106] S.A. Wolpert. On the Weil-Petersson geometry of the moduli space of curves. Amer. J. Math. 107 (1985), 969-997. MR 87b:32040

[107] S.A. Wolpert. Thurston's Riemannian metric for Teichmüller space. J. Diff. Geom. 23 (1986), 143-174. MR 88e:32035

[108] S.A. Wolpert. Geodesic length functions and the Nielsen problem. J. Diff. Geom. 25 (1987), 275-296. MR 88c:32032

[109] R.L. Zimmer. Ergodic theory and semisimple groups. Birkhäuser (1985). MR 86j:22014

Institut de mathématiques, Université de Neuchâtel, Rue Emile Argand 11, CH-2007 Neuchâtel, Switzerland

E-mail address: Paul.Schmutz@maths.unine.ch 